Artigo Original

\title{
Alterações nas variáveis de saúde em mulheres participantes de um programa público de exercícios físicos
}

\author{
Kaimi Pereira Costa ${ }^{1}$ \\ Carlos Eduardo Cesar Flores ${ }^{2}$ \\ Cândido Simões Pires Neto ${ }^{3}$ \\ ${ }^{1}$ Secretaria Municipal da Saúde de Curitiba - SMS/PMC, PR, Brasil \\ ${ }^{2}$ Universidade Federal do Paraná - UFPR, PR, Brasil \\ ${ }^{3}$ Unidade de Ensino Superior Vale do Iguaçu - UNIGUAÇU, PR, Brasil
}

\begin{abstract}
Resumo: No envelhecimento as alterações nos indicadores de desempenho e magreza-obesidade acarretam efeitos deletérios à saúde e que limitam a funcionalidade em humanos. Objetivou-se analisar as eventuais alterações nos indicadores de saúde em mulheres inseridas em programa público de exercícios físicos. Dezoito mulheres participaram durante sete meses em aulas de 50 min no Centro de Esporte e Lazer Vila Oficinas de Curitiba, PR. Analisou-se: massa corporal, estatura, IMC, RCQ, \%G, resistência muscular abdominal (RMA), flexibilidade, aptidão cardiorrespiratória, ( $\left.\mathrm{VO} 2_{\text {máx }}\right)$ e frequência cardíaca em repouso (FCR). Utilizou-se a estatística descritiva e teste t de Student $(p \leq 0,05)$. Os resultados indicaram que, exceto a idade e a FCR, as demais variáveis não foram alteradas significantemente $(p>0,05)$ entre avaliações. Conclui-se que as atividades desenvolvidas melhoram o sistema cárdiorespiratório pela diminuição da $\mathrm{FCR}$ e incremento do $\mathrm{VO}_{2}$ e acompanhadas pelas reduções da $\mathrm{MC}$ e IMC, embora a RMA, a $\mathrm{RCQ}$ e a flexibilidade tenham permanecido estáveis.
\end{abstract}

Palavras-chave: Antropometria. Aptidão física. Frequência cardíaca.

\section{Alterations on health variables of women engaged in a public physical exercise program}

\begin{abstract}
The ageing process produce deleterious effects upon health, performance and leanness-obesity which in turn it restrain human functionality. This study analyzed eventual effects of seven months of exercises upon health indicators of women involved at a physical exercise public program. During seven months, 18 women participated in 50 min exercise activities (3x week), at Centro de Esporte e Lazer (Center for Sports and Leisure) Vila Oficinas, Curitiba, PR. Body mass, stature, BMI, WHR, \%fat, abdominal strength (AS), flexibility (FLEX), cardiorespiratory fitness (CRF), estimated $\mathrm{VO}_{2 \max }$ and resting heart rate (RHR) were compared. Statistics consisted of descriptive and Student's dependent $t$ test, $p \leq 0.05$. Results showed that only RHR and age were significantly altered. It can be concluded that physical activities were effective to promote a RHR reduction and a $\mathrm{VO}_{2}$ increment both followed by a lower BM and BMI values, although AS, WHR, and flexibility remained steady.
\end{abstract}

Key Words: Anthropometry. Physical fitness. Heart rate.

\section{Introdução}

A urbanização acelerada e mudanças sóciodemográficas advindas do crescimento populacional e tecnológico das ultimas décadas tem interferido e modificado profundamente $o$ comportamento humano nas grandes cidades em decorrência, possivelmente, do aumento do tempo de deslocamento entre a residência e o trabalho além da dupla jornada de atividades. Conforme o ACSM (2006) e SAWAF \& CASSOU (2005), tanto nas nações desenvolvidas como nos países em desenvolvimento, existe uma grande escassez de áreas livres e seguras para a prática de atividades físicas. Por consequência, cada vez mais as pessoas restringem-se aos seus espaços, o que tem contribuído para o aumento do sedentarismo e ao aumento do risco do aparecimento das chamadas doenças crônicodegenerativas.

Para minimizar essa premissa algumas estratégias políticas em diversos países, inclusive no Brasil, foram e estão sendo implementadas com o intuito de conscientizar e aumentar os níveis de atividade física populacional (HALLAL \& CARVALHO, 2006; SAWAF \& CASSOU, 2005), por sua vez diminuindo os gastos com a saúde pública. A Secretaria Municipal de Esporte e Lazer da cidade de Curitiba (PR) iniciou em 1998 o programa CuritibAtiva. Este programa tem como filosofia, vocação e política de ação, a 
democratização das práticas desportivas e de lazer na cidade, oferecido de forma gratuita nos mais de trinta Centros de Esporte e Lazer, praças, parques, piscinas e academias, espalhadas nas nove regiões que dividem a metrópole. O programa ainda criou uma rede de atenção à população, pela informação, sensibilização, incentivo e ofertas da prática da atividade física, nos diferentes segmentos e nas diferentes faixas etárias, para mudança de atitudes e hábitos saudáveis e com isto melhorar a qualidade de vida.

Considerando que o processo de envelhecimento é acompanhado pelos aumentos nas massas de gordura e corporal, diminuição gradativa da estatura, do consumo máximo de oxigênio, $\mathrm{VO}_{2 \max }$, (potencia aeróbia), da massa muscular esquelética e, por conseqüência, na força muscular (MATSUDO; MASTUDO; BARROS, 2000). Portanto, torna-se importante determinar essas variações em indivíduos praticantes de exercícios físicos orientados, pois nessas pessoas essas modificações parecem ser menores em relação aos indivíduos sedentários.

Dessa forma o objetivo dessa pesquisa foi analisar as eventuais alterações em sete meses nos indicadores de saúde $\left(\mathrm{VO}_{2}\right.$, frequência cardíaca em repouso, IMC, \%G, flexibilidade e resistência muscular) em mulheres inseridas em um programa público de exercícios físicos.

\section{Procedimentos Metodológicos}

\section{Caracterização da amostra}

Este estudo se caracteriza como descritivo e quase-experimental (Thomas e cols, 2007, p. 290). Para a elaboração desse estudo seguiu-se as normas estabelecidas pela CONEP, Resolução No. 196/96, sobre pesquisas envolvendo seres humanos. Foram analisados somente os dados de 18 mulheres que cumpriram com os requisitos de freqüência entre o pré e pós-teste durante os sete meses. 0 numero de avaliadas é uma limitação do estudo. Contudo, inicialmente o grupo foi formado por 86 mulheres entre 36 e 59 anos de idade, todas participantes de um programa livre de exercícios físicos oferecido pela Secretária Municipal de Esporte e Lazer, da Prefeitura de Curitiba, PR. A amostra voluntária, $\mathrm{N}=18$, como acima explicado, foi escolhida intencionalmente no Centro Municipal de Esporte e Lazer Vila Oficinas, da capital paranaense.
O programa de exercícios físicos realizado por sete meses, 3 vezes por semana e com duração de 50 minutos por sessão incluía atividades para flexibilidade, exercícios aeróbios e resistidos, orientados por um profissional de educação física. As atividades desenvolvidas seguiram a sequência indicada acima e eram diversificadas para manter a aderência ao programa.

\section{Procedimentos para coleta de dados}

A coleta de dados foi realizada no Centro Municipal de Esporte e Lazer da Vila Oficinas da cidade de Curitiba, PR. Sendo a primeira avaliação (pré-teste) realizada no mês de maio de 2007 e a segunda (pós-teste) realizada no mês de novembro do mesmo ano. Utilizou-se uma ficha de registro na seguinte ordem: nome, idade, massa corporal, estatura, índice de massa corporal, perímetro da cintura, perímetro do quadril, relação cintura quadril, percentual de gordura, freqüência cardíaca em repouso e indicadores da aptidão física relacionada à saúde (aptidão cardiorrespiratória, flexibilidade e resistência muscular abdominal).

$\mathrm{Na}$ coleta dos dados da massa corporal (MC, $\mathrm{kg}$ ) foi utilizada uma balança digital, com capacidade para $150 \mathrm{~kg}$ e resolução de $100 \mathrm{~g}$, marca PLENNA. Na mensuração da estatura (EST, m) utilizou-se um estadiômetro, marca STANDARD, e resolução de $1 \mathrm{~mm}$. Para a massa corporal e estatura seguiu-se os critérios, orientações e recomendações de Alvarez e Pavan (2007). Para o IMC $\left(\mathrm{kg} / \mathrm{m}^{2}\right)$ as classificações utilizadas foram as da WHO, 1998. Os perímetros foram medidos com uma fita métrica inextensível de fibra de vidro, com dois metros e com resolução de $1 \mathrm{~mm}$, pelos procedimentos de Callaway et al. (1988) e as normas de Bray e Gray (1988) para a relação cintura quadril $(R C Q, c m)$. Para o calculo do percentual de gordura $(\% \mathrm{G})$ utilizou-se a equação proposta por Deurenberg et al.(1991) onde $\% \mathrm{G}=$ $1,20^{*}(\mathrm{IMC})+0,23^{*}$ (idade) $-10,8^{*}($ sexo $)-5,4$, sendo o sexo $=0$ para as mulheres, sendo uma limitação do estudo.

Para coleta de dados da freqüência cardíaca de repouso (FCR, bpm) utilizou-se um relógio frequêncimetro, da marca POLAR modelo $(\mathrm{S} / 210)$, estando a avaliada sentada por um período entre 5 a 10 minutos. Para a mensuração da FC em repouso seguiu-se o ACSM (2006). 
Para a avaliação da capacidade cardiorrespiratória aplicou-se o teste de $12 \mathrm{~min}$. utilizando-se de uma pista de 100 metros devidamente demarcada a cada 5 metros, de maneira que o numero de voltas completadas poderia ser facilmente contado e multiplicado pela distancia do percurso. As normas para a classificação seguiram Cooper (1968). Depois de calculada a distancia percorrida ao termino do teste de andar-correr de $12 \mathrm{~min}$, estimou-se o consumo de oxigênio através da formula $\mathrm{VO}_{2 \text { máx }}=$ 0,0268 (distância, m) - 11,3 proposta pelo autor (COOPER, 1968). O uso desta técnica é uma limitação do estudo.

A flexibilidade foi mensurada através do banco KR (adaptado do Banco de Wells), desenvolvido por Kruchelski e Rauchbach (2005), com dimensões de $30 \times 30 \mathrm{~cm}$, dotado de uma régua fixa, recuada a $15 \mathrm{~cm}$ do local de apoio dos pés. A adaptação do banco KR consistiu em deixar mais alta a peça móvel de madeira de maneira a permitir que o avaliado, sentado, encostasse a cabeça, a coluna vertebral e o quadril em uma parede ou apoio, formando um ângulo de $90^{\circ}$ com a articulação do quadril, estendendo os braços à frente, paralelos ao chão e encostasse a ponta dos dedos das mãos na peça de madeira móvel, sem perder o contato com a parede ou apoio, permitindo que todas as pessoas iniciassem o teste a partir do seu próprio referencial. A melhor de três tentativas foi adotada com referencia. O protocolo realizado na avaliação da resistência muscular abdominal seguiu Pollock e Wilmore (1993) sendo anotado o número de exercícios abdominais, realizados em 1 minuto.

\section{Tratamento estatístico}

Para analise das variáveis deste estudo utilizou-se a estatística descritiva e o teste $t$ pareado de Student sendo $p \leq 0,05$. Os cálculos foram realizados pelo software SPSS 13.0 for Windows.

\section{Resultados}

Pode-se constatar, por meio do teste $t$ pareado de Student (tabela 1), que em um período de sete meses, do pré-teste para o pós-teste, ocorreram alterações significativas $(p \leq 0,05)$ na idade entre o pré e pós-teste. Não ocorreram alterações significantes nas variáveis da MC, EST, IMC, RCQ e no \%G. A estabilidade dos valores da estatura pode ser atribuída ao melhor alinhamento da coluna vertebral (postura).

A tabela 1 indica que a média da idade aumentou em 0,5 meses. A média da $M C$, tabela 1 , teve um decréscimo de $1 \mathrm{~kg}$ ou $1,53 \%$. A média da EST permaneceu sem alterações no pós-teste. A média IMC (tabela 1) diminuiu em $0,71 \%$ ou seja, $0,2 \mathrm{~kg} / \mathrm{m}^{2}$. Os valores médios da RCQ permaneceram sem alterações do pré o para pós-teste e o $\% \mathrm{G}$ diminui em $0,3 \%$.

Tabela 1. Valores médios das variáveis idade, MC, EST, IMC, RCQ, \%G, teste t pareado e probabilidade.

\begin{tabular}{|c|c|c|c|c|c|c|c|c|c|c|c|c|}
\hline Variáveis & ID1 & ID2 & MC1 & MC2 & EST1 & EST2 & IMC1 & IMC2 & RCQ1 & RCQ2 & \%G 1 & \%G2 \\
\hline Média & 50,55 & 51,05 & 65,10 & 64,65 & 1,52 & 1,52 & 28,03 & 27,83 & 0,89 & 0,89 & 39,86 & 39,74 \\
\hline DP & 5,62 & 5,66 & 10,12 & 9,61 & 0,06 & 0,06 & 3,97 & 4,03 & 0,07 & 0,08 & 5,01 & 4,98 \\
\hline Min. & 36,00 & 36,00 & 53,60 & 54,00 & 1,42 & 1,42 & 21,91 & 22,35 & 0,79 & 0,77 & 30,76 & 31,15 \\
\hline Máx. & 59,00 & 59,00 & 86,20 & 83,70 & 1,64 & 1,65 & 35,37 & 36,23 & 1,02 & 1,03 & 49,69 & 48,43 \\
\hline Dif \% & \multicolumn{2}{|c|}{$+0,98$} & \multicolumn{2}{|c|}{$-0,69$} & \multicolumn{2}{|c|}{0,0} & \multicolumn{2}{|c|}{$-0,71$} & \multicolumn{2}{|c|}{0,0} & \multicolumn{2}{|c|}{$-0,30$} \\
\hline $\mathrm{t}$ & \multicolumn{2}{|c|}{ - 4,123 } & \multicolumn{2}{|c|}{0,723} & \multicolumn{2}{|c|}{$-1,000$} & \multicolumn{2}{|c|}{0,709} & \multicolumn{2}{|c|}{$-0,040$} & \multicolumn{2}{|c|}{0,362} \\
\hline $\mathrm{P}$ & \multicolumn{2}{|c|}{$0,001^{*}$} & \multicolumn{2}{|c|}{0,480} & \multicolumn{2}{|c|}{0,331} & \multicolumn{2}{|c|}{0,488} & \multicolumn{2}{|c|}{0,969} & \multicolumn{2}{|c|}{0,722} \\
\hline
\end{tabular}

*Diferença significativa, $\mathrm{p} \leq 0,05$; os valores 1 e 2, após cada sigla nas tabelas indicam pré e pós-testes, respectivamente.

A tabela 2 indica que, após sete meses de exercícios físicos orientados, ocorreram alterações significativas $(p \leq 0,05)$ na freqüência cardíaca de repouso $(F C R)$. O mesmo não aconteceu de forma significante $(p>0,05)$ na distância percorrida no teste de 12 min no consumo máximo de oxigênio estimado, na flexibilidade e na resistência muscular abdominal. 
Tabela 2. Valores médios das variáveis de FCR, $12 \mathrm{~min}, \mathrm{VO}_{2}$, FLEX, RMA, teste t pareado e probabilidade.

\begin{tabular}{|c|c|c|c|c|c|c|c|c|c|c|}
\hline Variáveis & FCR1 & FCR2 & $12 \min 1$ & $12 \min 2$ & $\mathbf{V O}_{2 \text { máx }} \mathbf{1}$ & $\mathrm{VO}_{2 \text { máx }} 2$ & FLEX1 & FLEX2 & RMA1 & RMA2 \\
\hline Média & 84,00 & 74,44 & 1309,61 & 1388,05 & 23,79 & 25,89 & 26,40 & 25,71 & 6,88 & 5,22 \\
\hline DP & 14,05 & 11,73 & 163,67 & 225,63 & 4,38 & 6,04 & 5,42 & 5,90 & 8,46 & 8,54 \\
\hline Min. & 64,00 & 52,00 & 975,00 & 1040,00 & 14,83 & 16,57 & 18,30 & 17,00 & 0,00 & 0,00 \\
\hline Max. & 112,0 & 100,0 & 1655,00 & 1970,00 & 33,05 & 41,50 & 39,20 & 38,00 & 28,00 & 29,00 \\
\hline Dif \% & \multicolumn{2}{|c|}{$-12,84$} & \multicolumn{2}{|c|}{$+5,65$} & \multicolumn{2}{|c|}{$+8,11$} & \multicolumn{2}{|c|}{$-2,68$} & \multicolumn{2}{|c|}{$-31,80$} \\
\hline $\mathrm{t}$ & \multicolumn{2}{|c|}{3,386} & \multicolumn{2}{|c|}{$-1,557$} & \multicolumn{2}{|c|}{$-1,557$} & \multicolumn{2}{|c|}{0,431} & \multicolumn{2}{|c|}{1,274} \\
\hline$P$ & \multicolumn{2}{|c|}{$0,004^{*}$} & \multicolumn{2}{|c|}{0,138} & \multicolumn{2}{|c|}{0,138} & \multicolumn{2}{|c|}{0,672} & \multicolumn{2}{|c|}{0,220} \\
\hline
\end{tabular}

*Diferença significativa, $p \leq 0,05$; os valores 1 e 2 , após cada sigla nas tabelas indicam, respectivamente, pré e póstestes.

\section{Discussão}

Considerando as elencadas limitações do estudo, observa-se que a redução da massa corporal em indivíduos acima do peso tem demonstrado efeitos positivos à saúde e um impacto significativo no tratamento da hipertensão arterial (ACSM, 2006). É evidente que a pratica regular de exercícios físicos, aliada à outras intervenções no estilo de vida tende a auxiliar na redução da $\mathrm{MC}$, embora Ross e Janssen (2001) afirmem que a redução na massa corporal torna-se mais aparente nas primeiras semanas de atividade, e que a partir de então, dependendo do volume e da intensidade, o exercício exerceria pouca influencia no decréscimo da MC. Assim como no presente estudo, Farinatti et al. (2005) observaram que, em quatro meses de um programa domiciliar de exercícios físicos, também encontraram redução na massa corporal.

A utilização dos indicadores antropométricos de IMC e da RCQ são comumente utilizados como marcadores de saúde sendo que níveis elevados nessas variáveis possuem fortes associações com o aparecimento de hipertrigliceridemia, comprometimento do metabolismo das lipoproteínas e surgimento de doenças coronarianas (GUIMARÃES \& PIRESNETO, 1997). Embora as variáveis do IMC e $R C Q$, no presente estudo (tabela 1), não tenham se modificado significativamente, $p>0,05$, permanecendo em sobrepeso e em risco muito alto a saúde, respectivamente, pode-se constatar que estas práticas regulares de exercícios físicos ( 3 vezes por semana) foram benéficos para uma leve melhoria e/ou manutenção dessas variáveis, o que é um fato destacável, pois inúmeros estudos correlacionam o aumento da idade cronológica com o acréscimo nesses índices.
Matsudo et al. (2004) ao avaliarem mulheres participantes de um programa de exercícios, similar ao desse relato, encontraram variações de menos de $1 \%$ no IMC e de menos de $2 \%$ na $R C Q$, em um período de 4 anos e indicaram que a evolução do perfil antropométrico de mulheres acima de 50 anos de idade, envolvidas regularmente em um programa de exercícios físicos, parece se manter estável durante o período de um ano, independente da idade cronológica e sugerem, para minimizar os efeitos deletérios do envelhecimento nas variáveis relacionadas à saúde, a adoção e manutenção de um estilo de vida ativo.

Lima e Glaner (2006) informam que independente da técnica utilizada para quantificar a topografia da gordura corporal, alguns estudos apontam que $\mathrm{o}$ acumulo de gordura, principalmente na região do tronco, explicam melhor as alterações nas concentrações sangüíneas de glicose, colesterol, triglicérides e outros fatores de risco, comparado a estimativa do percentual de gordura por si só. Analisando o $\%$ G (tabela 1) pode-se observar do pré para o pós-teste que o percentual permaneceu elevado, embora tendo uma pequena diminuição de $0,3 \%$ mostrando que as atividades desenvolvidas, não foram de intensidade suficiente para promover alterações significantes nesta variável, entretanto, o desvio padrão menor no pós-teste indica uma menor heterogeneização dos resultados. Venturim e Cade (2007) objetivando conhecer o efeito da prática de atividade física e de orientação dietética sobre as variáveis antropométricas, bioquímicas e hemodinâmicas, evidenciaram um decréscimo de $6,9 \%$ e $9,5 \%$ no $\% \mathrm{G}$ em um período de quatro e onze meses, respectivamente. Resultado semelhante foi encontrado por Prestes et al. (2006) em mulheres 
entre 35 e 55 anos, participantes de três sessões semanais de exercícios aeróbio e de força, durante 16 semanas, onde foi relatada a redução de 3\% na G\% após esse período.

Segundo Almeida (2007) a freqüência cardíaca tem sido utilizada como uma das principais variáveis fisiológicas relacionadas ao controle e prescrição do exercício físico. O mesmo autor afirma que a redução da FCR é uma adaptação crônica comum após um período de treinamento aeróbio e, de forma menos significativa, após o treinamento de força. $\mathrm{Na}$ comparação das respostas hemodinâmicas de repouso Abreu et al. (2007) encontraram diminuições maiores na FCR, após 3 meses de treinamento resistido quando combinado com o treinamento aeróbio. Especula-se que quanto maior a oscilação da FCR, após um período de treinamento, maior a participação do ramo parassimpático (atividade vagal cardíaca). Embora não exista ainda uma medida padronizada para a mensuração da FCR ela tende a refletir uma condição saudável, ou seja, indivíduos com menor FCR ou menor FC durante o exercício apresentam menor probabilidade de desenvolverem cardiopatias (ALMEIDA, 2007). Pode-se verificar (tabela 2) que a FCR teve uma redução importante e significativa $(p \leq 0,05)$ após sete meses de atividades físicas, com uma diminuição de 9,56 batimentos por minuto (bpm). A redução significante na FCR em mulheres, após um período de treinamento físico (11 meses), também foi relatada por Venturim e Cade (2007).

Os testes cardiorrespiratórios são importantes no planejamento do treinamento físico, no diagnostico, na promoção de saúde e por não requerer o uso de equipamentos sofisticados e um alto grau de controle, é inegável a praticidade da aplicação do teste de campo de caminhar, trotar e/ou correr de $12 \mathrm{~min}$, principalmente quando empregado em grandes amostras (SPECK et al., 2007). Glaner (2003) informa que são inúmeros os estudos que evidenciaram que indivíduos bem treinados aerobicamente apresentam menor risco de doença coronariana, acidente vascular cerebral, diabetes, hipertensão arterial, vários tipos de câncer, obesidade, osteoporose, ansiedade e depressão. Os resultados encontrados no presente estudo (tabela 2) indicam que apesar da melhoria do pré para o pós-teste de $78,44 \mathrm{~m}$ na distância percorrida no teste de $12 \mathrm{~min}$, os sete meses de exercícios físicos não foram suficientes para promoção de ganhos significativos nessa variável, indicando que aspectos como a especificidade, intensidade e volume do treinamento necessitam de maior controle, embora os exercícios físicos desenvolvidos foram benéficos para promover uma redução significante, $p \leq 0,05$, na FCR.

Além da distância percorrida no teste de 12 min, um dos objetivos do estudo foi verificar as alterações no consumo máximo estimado de oxigênio $\left(\mathrm{VO}_{2 m a ́ x}\right)$ pois cientistas do exercício o consideram como o indicador mais válido da capacidade funcional do sistema cardiorrespiratório (ACMS, 2006). Embora do pré para o pós-teste o $\mathrm{VO}_{2 m a ́ x}$ (tabela 2 ) não tenha se alterado significativamente $(p>0,05)$, as atividades físicas o aprimoraram em $8 \%$, fato importante para as avaliadas, haja visto que o processo de envelhecimento é acompanhado por uma perda da potencia aeróbia em torno de $1 \%$ ao ano (MATSUDO; MATSUDO; BARROS, 2000). Aumento similar no $\mathrm{VO}_{2 \text { máx }}$, encontrado no presente estudo, também foi relatado por Rossato et al. (2007) que observaram uma melhoria de $7,23 \%$ no $\mathrm{VO}_{2 \text { máx }}$ após 20 semanas de treinamento de força e de endurance, em mulheres na fase perimenopausa. Para ganhos mais significativos no $V_{2 m a ́ x}$ pesquisas demonstraram (VAN AGGEL-LEIJSSEN et al., 2002; KEMMLER et al., 2005) que a combinação prolongada de exercícios aeróbios, exercícios resistidos, orientações nutricionais e a prescrição da atividade em torno de 70 a $80 \%$ do $\mathrm{VO}_{2 \text { máx }}$ tendem a ser mais eficazes.

Em relação à repetitividade dos movimentos domésticos e laborais diários da mulher, Theodoro e Gagliardi (2005) comentam que é inevitável a perda de amplitude de movimento articular se não houver paralelamente um trabalho de manutenção e/ou aumento da flexibilidade. No presente relato (tabela 2), podese verificar que o programa de exercícios não foi benéfico para aumento da flexibilidade (FLEX), contudo as avaliadas permaneceram classificadas no nível razoável (KRUCHELSKI \& RAUCHBACH, 2005). Este feito é relevante pois, o aumento da idade cronológica é seguido de uma diminuição gradativa no desempenho neuromotor e nos casos de problemas relacionados à coluna (lombalgias, ciatalgias, 
escolioses) uma boa flexibilidade mostra-se como um importante fator de contribuição no equilíbrio músculo-esquelético, reduzindo as dores provenientes de um desequilíbrio entre força e alongamento muscular, colaborando ainda para um melhor bem-estar, diminuindo riscos de lesões, além de facilitar nas atividades do cotidiano (MATSUDO; MATSUDO; BARROS, 2000; GLANER, 2003; ALMEIDA; JABUR, 2007). Reforçando os achados do atual relato, Matsudo et al. (2003) também não encontraram diferenças significativas $(p>0,05)$, ao longo de 1 ano, na flexibilidade de mulheres que realizaram exercícios aeróbios por duas vezes na semana.

Quando o componente da aptidão física relacionada à saúde foi analisado, verificou-se que os efeitos da resistência muscular abdominal (RMA) (tabela 2) estão de acordo com as alterações na aptidão física resultantes do processo de envelhecimento, onde, em decorrência da atonia muscular ocorre um decréscimo gradativo da força muscular (MATSUDO, MATSUDO e BARROS, 2000), o que pode implicar em dificuldades nas atividades do cotidiano pois, em sua maioria, essas pessoas, necessitam de mais resistência muscular, o que está em contraste ao desempenho obtido por mulheres mais jovens, treinadas ou não, em distintos testes de RMA, segundo o relato de Moura, Pedroso e Zinn, (2002).

\section{Conclusão}

Conforme as limitações do estudo e de acordo com os resultados obtidos pode-se concluir que o programa público de exercícios físicos combinando flexibilidade, atividades aeróbias e exercícios resistidos, durante um período de sete meses, foram suficientes para promover alterações significativas na FCR, enquanto que nas variáveis de MC, EST, IMC, RCQ, \%G, 12 min e $V_{2 m a ́ x}$ observou-se uma tendência de manutenção ou pequenas melhorias nos índices alcançados, fato destacável haja visto as mudanças ocorridas na composição corporal e na resistência aeróbia com o avanço etário. $\mathrm{Na}$ flexibilidade e na resistência muscular abdominal ocorreram pequenos decréscimos após os sete meses de praticas de atividades físicas, sugerindo que um período de pratica maior e/ou especificidade no treinamento poderia ser necessário para melhorias nessas variáveis.

\section{Referências}

ABREU, D. G.; GOMES, A. L. M.; MOTA, R. et al. Reabilitação cardíaca: comparação das respostas hemodinâmicas de repouso entre exercícios contra resistivos e exercícios contra resistivos conjugados com exercícios aeróbicos em indivíduos cardiopatas. Revista Treinamento Desportivo, Curitiba, v.8, n.1, p.45-52, 2007. http://www.treinamentodesportivo.com.br/artigos/ 08 Artigo VOL 8 N1 $2007 \mathrm{Pg} 45$ a 52. Acesso em: 25 de mai. 2008.

ALMEIDA, M. B. Freqüência cardíaca e exercício: uma interpretação baseada em evidências.

Revista Brasileira de Cineantropometria \& Desempenho Humano, Florianópolis, v.9, n.2, p.196-202, 2007.

http://www.periodicos.ufsc.br/index.php/rbcdh/arti cle/ view /4063/3438. Acesso em 03 de mai. 2008.

ALMEIDA, T. T.; JABUR, N. M. Mitos e verdades sobre flexibilidade: reflexões sobre o treinamento de flexibilidade na saúde dos seres humanos. Motricidade, Santa Maria da Feira, v.3, n.1, p.337-344, 2007.

http://www.scielo.oces.mctes.pt/pdf/mot/v3n1/v3n 1a08.pdf. Acesso em 12 de jul. 2008.

ALVAREZ, B. R.; PAVAN, A. L. Alturas e comprimentos. In PETROSKI, E. L.

Antropometria Técnicas e Padronizações. Blumenau, SC: Nova Letra, 2007.

AMERICAN COLLEGE OF SPORTS MEDICINE - ACSM'S. Guidelines for Exercise Testing and Prescription. Philadelphia: Lippincott Williams \& Wilkins, $7^{\mathrm{a}}$ ed, 2006.

BRAY, G. A.; GRAY, D.S. Obesity. Part I Pathogenesis. Western Journal of Medicine. 149: p. 429-441, 1988 b.

CALLAWAY, C. W.; CHUMLEA, W. C.; BOUCHARD, C. et al. Circunferences. In: LOHMAN, T. G.; ROCHE, A. F.; MARTOREL, R. Anthropometric Standardization Reference Manual. Champaign, Illinois: Human Kinetics Books, 1988.

COOPER, K, H. A means of assessing maximal oxygen intake: correlation between field and treadmill testing. JAMA v.203, p.135-138, 1968.

DEURENBERG, P.; WESTSTRATE, J. A.; SEIDELL, J. C. Body mass index as a measure of body fatness: age- and sex specific prediction formulas. British Journal of Nutrition. v.65, p.105-114, 1991. 
FARINATTI, P. T. V.; OLIVEIRA, R. B.; PINTO, V. L. M.; MONTEIRO, W. D.; FRANCISCHETTI, E. Programa domiciliar de exercícios: efeitos de curto prazo sobre a aptidão física e pressão arterial de indivíduos hipertensos. Arquivos Brasileiros de Cardiologia, Rio de Janeiro, v.84, n.6, p.473-479, 2005.

http://www.arquivosonline.com.br/2005/8406/8406 0009.pdf. Acesso em 03 de mar. 2008.

GUIMARÃES F.J.S.P, PIRES-NETO C.S. Caracterização antropométrica e da composição corporal e suas relações com as doenças degenerativas. Corporis, v. 2, p. 23-29, 1997.

GLANER, M. F. Importância da aptidão física relacionada à saúde. Revista Brasileira de Cineantropometria \& Desempenho Humano, Florianópolis, v.5, n.2, p.75-85, 2003. http://www.periodicos.ufsc.br/index.php/rbcdh/arti cle/view/3963/3364. Acesso em: 30 mar. 2008.

HALLAL, P.C.; CARVALHO, Y. M. Avaliação de programas de atividade física voltados para a promoção da saúde: os casos "Curitibativa" e "Academia da Cidade". Projeto de Pesquisa. Fundação Delfim Mendes Silveira UFPel.2006.

KEMMLER, W.; VON STENGEL, S.; WEINECK, J.; et al. Exercise effects on menopausal risk factors of early postmenopausal women: 3-yr Erlangen fitness osteoporosis prevention study results. Medicine \& Science in Sports \& Exercise, v.37, n.2, p.194-203, 2005.

KRUCHELSKI, S., RAUCHBACH Avaliação da flexibilidade - adaptação para o teste de sentar e alcançar aplicada aos diferentes biotipos - estudo piloto. Ação \& Movimento, Rio de Janeiro, v. 2, n. 5, p.249-255, 2005.

LIMA W.A, GLANER M.F. Principais fatores de risco relacionados às doenças cardiovasculares. Revista Brasileira de Cineantropometria \& Desempenho Humano, Florianópolis, v.8, n.1, p.96-104, 2006.

http://www.periodicos.ufsc.br/index.php/rbcdh/arti cle /view /3770/3214. Acesso em: 07 nov. 2008.

MATSUDO, S. M.; MATSUDO, V. K.; BARROS, T. L. Impacto do envelhecimento nas variáveis antropométricas, neuromotoras e metabólicas da aptidão física. Revista Brasileira de Ciência e Movimento, Brasília, v.8, n.4, p. 21-32, 2000. http://www.ucb.br/mestradoef/RBCM/8/8\%20\%204/completo/c 84 3.pdf. Acesso em: 26 jul. 2008.

MATSUDO, S. M.; MATSUDO, V. K. R.; BARROS NETO, T. L.; ARAÚJO, T. L. Evolução do perfil neuromotor e capacidade funcional de mulheres fisicamente ativas de acordo com a idade cronológica. Revista Brasileira de Medicina do Esporte. v.9, n.6, p.365-376, 2003.

MATSUDO, S.M.; MARIN, R. V.; FERREIRA, M. T.; ARAUJO, T. L. Estudo longitudinal- tracking de 4 anos - da aptidão física de mulheres da maioridade fisicamente ativas. Revista Brasileira de Ciência e Movimento, Brasília, v.12, n.3, p.47-52, 2004.

MOURA, J. A. R.; PEDROSO, N. M. L.; ZINN, J. L. Avaliação da resistência muscular da região abdominal em clientes de academias de ginástica. Revista Brasileira de Cineantropometria \& Desempenho Humano, Florianópolis, v.4, n.2, p.46-52, 2002. http://www.periodicos.ufsc.br/index.php/rbcdh/arti cle/view/3980/3379. Acesso em 10 de ago. 2008.

POLLOCK, M. L.; WILMORE, J. H. Exercício na saúde e na doença: avaliação e prescrição para prevenção e reabilitação. 2 ed. São Paulo, SP: MEDSI, 1993.

PRESTES, J.; FROLLINI, A.B.; BORIN, J. P.; et al. Efeitos de um treinamento de 16 semanas sobre a composição corporal de homens e mulheres. Revista Brasileira de Atividade Física \& Saúde, Londrina, v.11, n.1, p.19-28, 2006.

ROSS, R.; JANSSEN, I. Physical activity, total and regional obesity: dose-response consideration. Medicine \& Science in Sports \& Exercise, Hagerstown, v.33, p.521-527, 2001.

ROSSATO, M.; BINOTTO, M. A.; ROTH, M. A.; et al. Efeito de um treinamento combinado de força e endurance sobre componentes corporais de mulheres na fase de perimenopausa. Revista Portuguesa de Ciências do Desporto, Porto, v.7, n.1, p.92-99, 2007. http://www.fade.up.pt/rpcd/arquivo/artigos soltos/ vol.7 nr.1/1.09.pdf. Acesso em 05 de fev. 2008.

SAWAF, C.; CASSOU, J. C. CuritibAtiva Programa de incentivo à atividade física da cidade de Curitiba. In: KRUCHELSKI, S.; RAUCHBACH, R. Organizadores. Curitiba Ativa gestão nas cidades voltada à promoção da atividade física, esporte, saúde e lazer. Curitiba;2005.

SPECK, L.M.; MACEDO, H. G. C.; CARVALHO, G.B.; et al. Comparação dos teste de Cooper e da Universidade de Montreal com o teste de medida direta de consumo máximo de oxigênio. Revista de Educação Física, Rio de Janeiro, v.136, p.1319, 2007.

http://www.revistadeeducacaofisica.com.br/ artigos/2007.1/testescooper.pdf. Acesso em abr. 2008. 
THEODORO, P. F. R.; GAGLIARDI, M. C. S.

Análise da flexibilidade em mulheres

trabalhadoras. Movimento \& Percepção, Espírito

Santo de Pinhal, v.5, n.7, p.116-123, 2005.

http://www.unipinhal.edu.br/movimentopercepcao/ viewarticle.php?id=46. Acesso em 20 abr. 2008.

THOMAS, J.R.; NELSON, J.K; SILVERMAN, S.J. Métodos de pesquisa em atividade física.

Porta Alegre: Artmed, 2007.

VAN AGGEL-LEIJSSEN, D. P. C.; SARIS, W. H. M.; WAGENMAKERS, A. J. M.; SENDEN, J. M.; VAN BAAK, M. A. Effect of exercise training at different intensities on fat metabolism of obese men. Journal of Applied Physiology, v.92, p.1300-1309, 2002.

VENTURIM, L. M. V. P.; CADÊ, N. V. Efeitos do programa "P.E.S.O" (Promoção de Estilo de Vida Saudável na Obesidade) sobre as variáveis antropométricas, hemodinâmicas e bioquímicas. Revista Brasileira de Atividade Física \& Saúde, Londrina, v.12, n.1, p.19-26, 2007.

WHO, Word Heath Organization. Obesity Status: preventing and managing the global epidemic. Report of a WHO Consultation on Obesity. Geneva, 1998.

\section{Endereço:}

Kaimi Pereira Costa

Manoel Borges, 130

Curitiba PR Brasil

82810-130

e-mail: kaimi8873@hotmail.com

\section{(ف) $\odot \Theta \Theta$}

Motriz. Revista de Educação Física. UNESP, Rio Claro, SP, Brasil - eISSN: 1980-6574 - está licenciada sob Licenca Creative Commons 